\title{
Malignant Rhabdoid Tumour of the Pineal Region
}

\author{
Matthew Muller, Sherri Lynn Hubbard, John Provias, Mark Greenberg, \\ Laurence E. Becker, and James T. Rutka
}

\begin{abstract}
A 9-month-old male presented to hospital with signs and symptoms of raised intracranial pressure. A CT scan showed obstructive hydrocephalus from a large pineal region mass lesion into which an intratumoral hemorrhage had occurred. A posterior fossa craniectomy and subtotal excision of the mass lesion were performed. By histopathology, the lesion was a malignant rhabdoid tumour (MRT). Despite surgery and chemotherapy, the tumour grew inexorably, and the patient died four months after the initial diagnosis. MRT is a rare and highly invasive neoplasm which infrequently arises from the central nervous system. This is the first documented case of a MRT arising from the pineal region. The clinical, radiographic, and pathological features of the MRT in this patient are presented.
\end{abstract}

Résumé: Tumeur rhaboïde maligne de la région pineale. Un bébé de sexe masculin, âgé de 9 mois, a été admis à l'hôpital parce qu'il présentait des signes et des symptômes d'hypertension intracrânienne. Un CT scan a montré une hydrocéphalie obstructive due à une grosse masse située dans la région pinéale dans laquelle une hémorragie intratumorale s'était produite. Par voie de la fosse postérieure, on a procédé à une excision subtotale de la masse. A l'histopathologie, la lésion était une tumeur rhabdoïde maligne (TRM). La tumeur a progressé inexorablement, malgré la chirurgie et la chimiothérapie, et le patient est décédé quatre mois après le diagnostic initial. La TRM est une néoplasie rare et très invasive originant peu fréquemment du système nerveux central. Ceci constitue le premier cas documenté d'une TRM originant de la région pinéale. Nous présentons les caractéristiques cliniques, radiologiques et pathologiques de la TRM chez ce patient.

Can. J. Neurol. Sci. 1994; 21: 273-277

The malignant rhabdoid tumour (MRT) is an uncommon neoplasm of early childhood. MRT is clinically characterized by an early age of onset and a poor prognosis. ${ }^{1.2}$ The high mortality associated with MRT results from its highly invasive and metastatic potential. The majority of MRTs occur in the infant kidney. MRT accounts for $2 \%$ of all childhood renal neoplasms. ${ }^{3}$ Extra-renal MRTs are much less common than renal MRTs. To date, only 13 cases of MRTs of the central nervous system (CNS) have been reported..$^{4-13}$ We present the 14 th documented case of a primary rhabdoid tumour of the CNS which satisfies the clinicopathological criteria for classification as a primary MRT. To our knowledge, this is the first reported case of a MRT arising from the pineal region. The clinical, radiographic and pathological findings of this rare but recently recognized CNS tumour are presented.

\section{CaSe Report}

A 9-month-old male infant presented to hospital with a 5 day history of vomiting and irritability. On examination, he was clinically dehydrated, lethargic and demonstrated a "sun-setting" gaze paresis. His head circumference measured $49 \mathrm{~cm}(>98$ th percentile) and his anterior fontanelle was full and tense. A CT scan showed enlarged ventricles and a large, hyperdense mass lesion in the pineal region which enhanced brightly but inhomogeneously following contrast administration
(Figure 1A \& B). Immediately following the CT scan, the patient's neurological condition deteriorated precipitously, and he required an urgent ventricular puncture for CSF which was followed by the insertion of a right VP shunt. To rule out a malignant germ cell tumour, CSF was taken at surgery and sent for cytology and tumour marker studies. The CSF was negative for malignant cells, and for alpha fetoprotein, human chorionic gonadotropin, carcinoembryonic antigen, and placental alkaline phosphatase. While he improved dramatically after his VP shunt, the next day the patient again became drowsy and bradycardic. A repeat CT scan showed that his ventricles were decompressed from the VP shunt, but that a large intratumoral hemorrhage had occurred (Figure 2). A suboccipital craniotomy was immediately performed, and a radical subtotal excision of the tumour was achieved. At surgery, the tumour was extremely vascular, and an intraoperative blood transfusion was required. Post-operatively, his neurological state was vastly improved, and aside from a transient right VIth nerve palsy, he made an uneventful recovery. A post-operative staging brain CT and spinal MRI were performed which showed that a small amount of residual tumour

From the Divisions of Neurosurgery (J.T.R.), Neuropathology (L.E.B., J.P.), Department of Oncology (M.G.), and Brain Tumour Research Laboratory (M.M., S.L.S), Hospital for Sick Children, University of Toronto, Toronto.

RECEIVED DECEMBER 1, 1993. ACCEPTED IN FINAL FORM MARCH 14, 1994.

Reprint requests to: James T. Rutka, Division of Neurosurgery, Suite 1502, Hospital for Sick Children, 555 Universtiy Avenue, Toronto, Ontario MSG IX8 
appeared confined to the pineal region (Figure 3) without evidence of neuraxis dissemination. A CT scan of the chest and abdomen was negative for mass lesions.

\section{Histopathologic Examination}

Tumour tissue obtained at surgery was fixed in $10 \%$ buffered formalin and embedded in paraffin for light and immunohistochemical microscopy. Portions of the tumour were also fixed in $2 \%$ glutaraldehyde with $0.1 \mathrm{M}$ phosphate-buffered osmium tetroxide, dehydrated in gradient ethanol, and embedded in Epon 812 for electron microscopy. Sections of the tumour revealed a very cellular malignant neoplasm

A
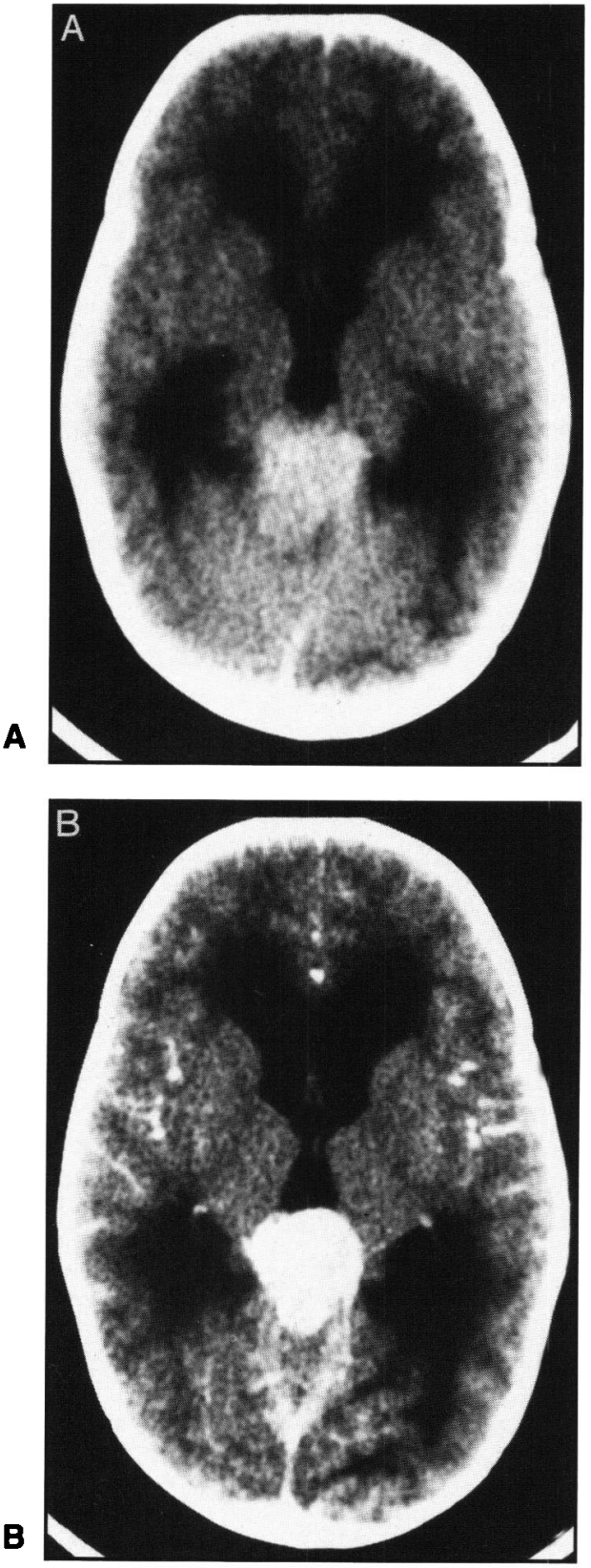

Figure 1: Plain A) and contrast-enhanced B) axial CT scans showing hyperdense pineal region mass which enhances inhomogeneously with contrast administration. Obstructive hydrocephalus with dilatation of the third and lateral ventricles is evident along with periventricular low density suggesting transependymal CSF flow from raised intracranial pressure. composed of sheets of round to polygonal epithelioid type cells. These cells contained large nuclei, many with prominent single, centrally placed nucleoli (Figure 4). Varying amounts of cytoplasm were present in the cells, with scattered cells showing well-defined perinuclear eosinophilic cytoplasm densities, some of which were hyaline-like in appearance (Figure 4). No areas of cytoplasmic striation were detectable. The tumour cells were closely crowded together, but were not truly cohesive. No cellular processes were evident. Nuclear pleomorphism and atypia with extremely abundant mitotic activity (2-4 mitoses per high powered field) were marked.

The tumour cells were strongly reactive with antiserum to vimentin and focally positive with antiserum to cytokeratin. Tumour cells reacted negatively to the following antisera: epithelial membrane antigen, actin, myoglobin, desmin, neuron specific enolase, neurofilament, synaptophysin, S100, Factor VIII, alpha-1-antitrypsin, leukocyte common antigen, and glial fibrillary acidic protein.

By electron microscopy, the cells were relatively undifferentiated, primitive appearing cells with a high nuclear : cytoplasmic ratio. The cells showed no junctional complexes. Nuclei showed a clumped chromatin pattern, with prominent nucleoli. Abundant perinuclear cytoplasmic masses of intermediate filaments were visible with focal areas of dense filamentous aggregation (Figure 5). Based on the above histopathological information, a diagnosis of malignant rhabdoid tumour of the pineal region was made.

\section{Post-operative Course}

The patient received courses of chemotherapy consisting of cycles of carboplatin, ifosphamide, and etoposide. However, over the course of the ensuing three months, a series of CT scans revealed rapid regrowth of the residual tumour mass, despite chemotherapy (Figure 6). The child once again deteriorated precipitously following a second intratumoural hemorrhage for which a repeat posterior fossa craniectomy was performed. The appearance of the tumour at pathological examination on this occasion was identical to the first examination. Following the second craniotomy, the patient continued to deteriorate, and died three and a half months after the initial craniotomy. An autopsy was not performed.

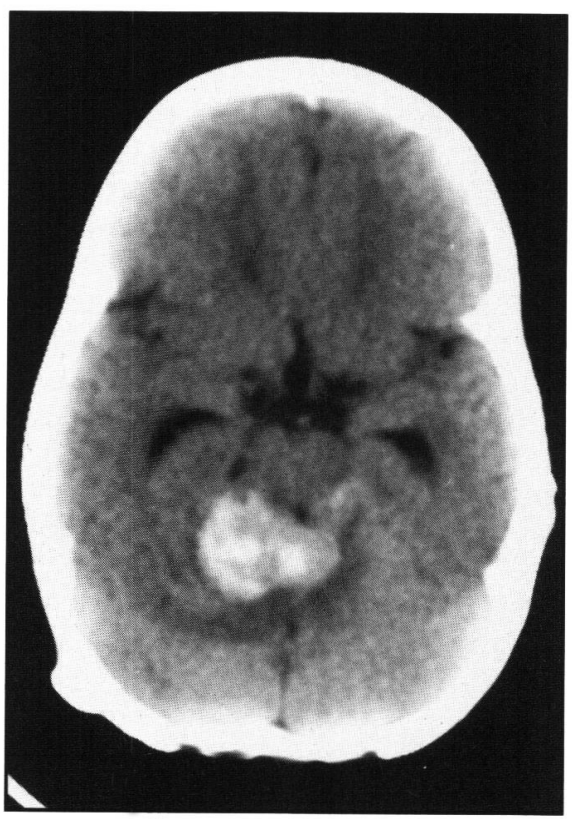

Figure 2: Repeat non-contrast CT scan following VP shunt insertion. The patient had deteriorated neurologically with bradycardia and depressed level of consciousness. A hyperdense region is seen within the tumour suggesting intratumoral hemorrhage. 


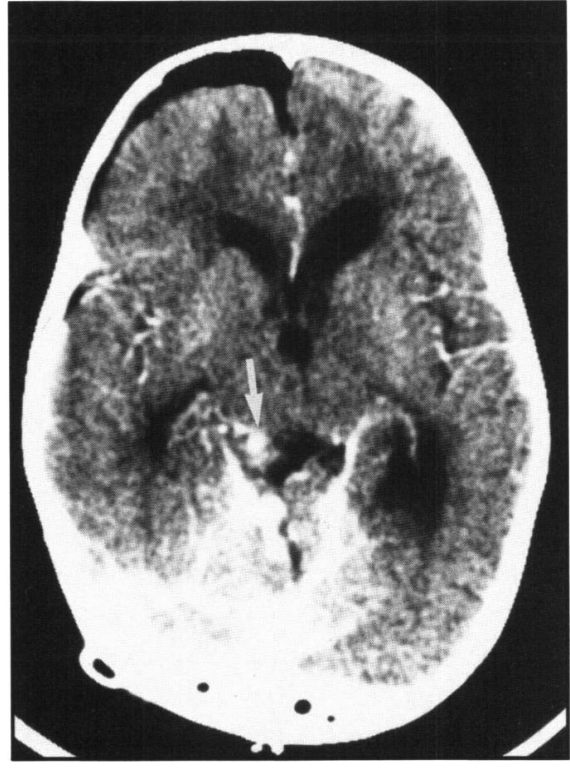

Figure 3: Immediate post-operative CT scan showing small amount of residual tumour in the right pineal region (arrows). The ventricles are smaller than pre-operatively, and the tumour has been largely excised.
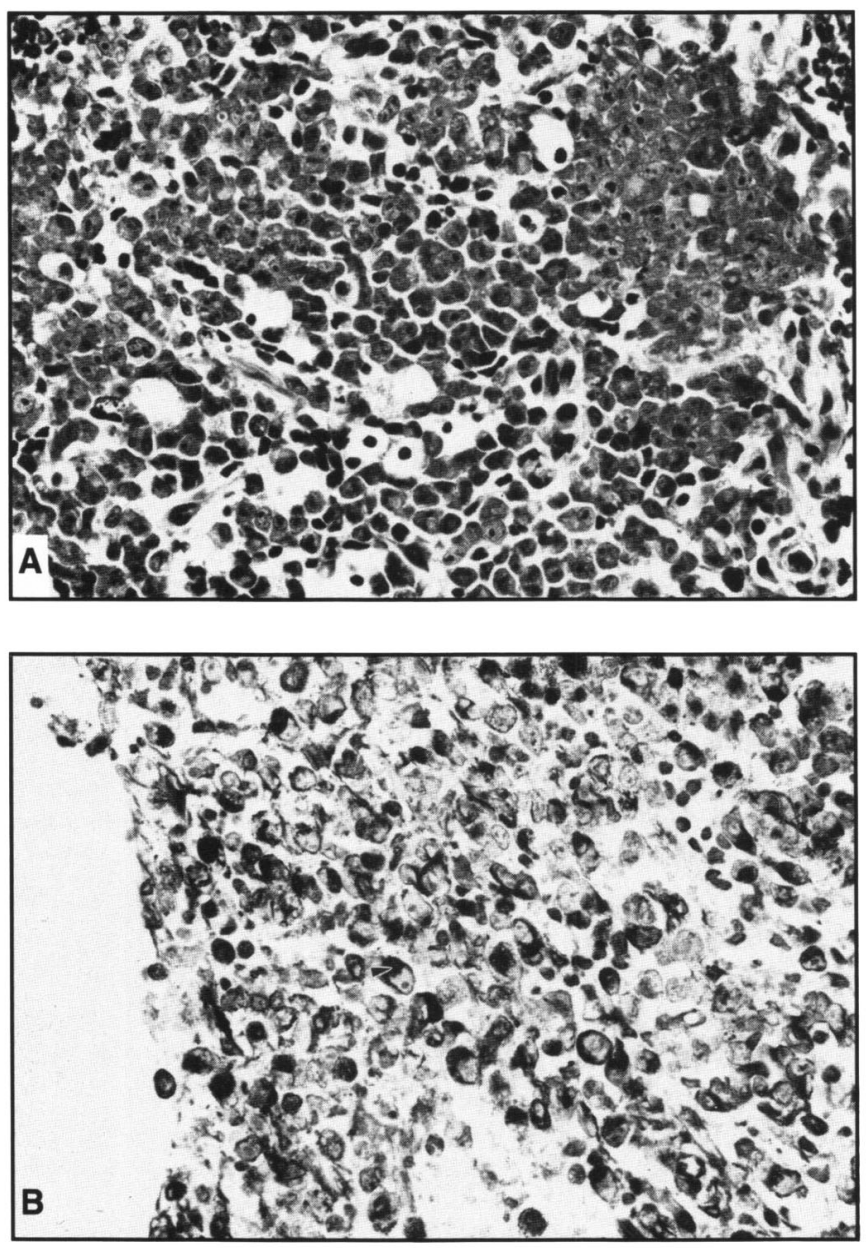

Figure 4: Histopathological examination of tumour shows: A) A hypercellular tumour with pleomorphic nuclei and prominent nucleoli. $H \&$ $E, x$ 400. B) Perinuclear eosinophilic cytoplasmic densities (arrow) immunoreactive with antiserum to vimentin. $H \& E, x 400$.

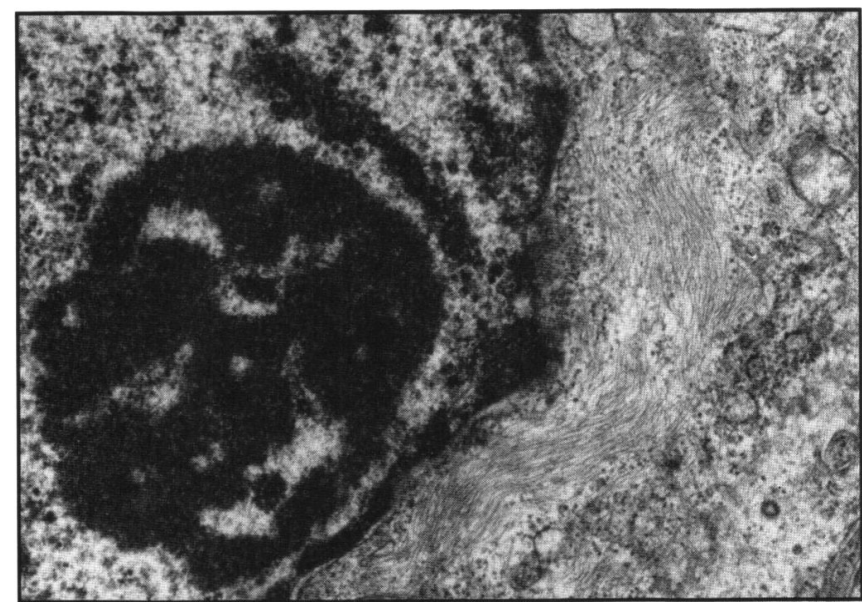

Figure 5: Ultrastructure of tumour cell, showing a prominent nuclolus and $a$ whorled mass of intermediate-sized filaments. TEM, $x 39,000$.

\section{Discussion}

This report documents a case of a primary MRT of the central nervous system, and the first reported case of a pineal region MRT. The diagnosis of primary CNS MRT was made from the histopathologic features of the tumour cells. MRT's may be recognized by several distinct features. Light microscopy reveals round or polygonal cells with single vesicular nuclei, single prominent nucleoli, and abundant eosinophilic cytoplasm. Ultrastructural features include whorls of intermediate filaments. Immunocytochemical stains are strongly positive for vimentin. Despite these characteristics, recognition of a true primary MRT is often difficult. Other neoplasms may display many of the features described above. In the case of an extrarenal MRT, the possibility of an undiagnosed kidney MRT which has metastasized must be considered. Computed tomography of the abdomen, chest and spine should be performed to ensure that the tumour is a primary neoplasm and not a metastasis. This is an important step in the diagnosis of primary MRT of the CNS, considering the highly metastatic nature of MRT.

Of the previously documented cases of central nervous system MRT, 5 were cerebellar in origin, ${ }^{6-8.10} 7$ were supratentorial, $4,5,11-13$ and one was multifocal. ${ }^{9}$ This case is the 8 th reported CNS MRT of supratentorial origin. This case shared many of the characteristic clinical features of other CNS rhabdoid tumours, including early onset, extremely aggressive and invasive behaviour, and near uniform fatality.

The most common treatment reported for CNS MRTs has been tumour debulking. In the 9 previous cases in which debulking was performed, ${ }^{4.5,8-12} 2$ total and 7 subtotal resections were reported. In our case, surgery was technically more difficult because of the the vascularity of the tumour. This should be kept in mind whenever surgery on these tumours is attempted. The extreme vascularity of these tumours may explain why our patient and the patient reported by Chou and Anderson ${ }^{10}$ presented with acute neurological deterioration from spontaneous intratumoural hemorrhaging.

The histogenesis of MRT of the CNS remains unknown as it does for rhabdoid tumours of the kidney. There are a number of morphologic and biologic similarities between CNS rhabdoid 
A
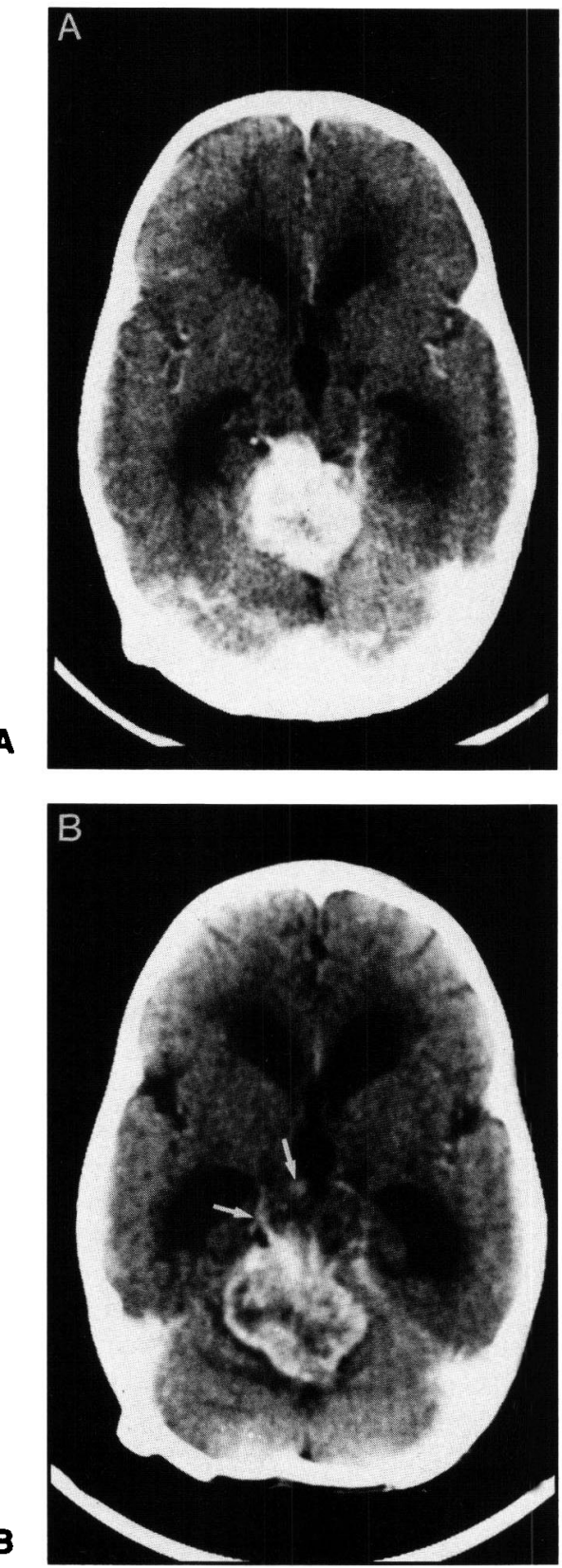

Figure 6: A) Post-chemotherapy contrast-enhanced axial CT scan 3 months after initial diagnosis showing rapid regrowth of the pineal region MRT. B) On lower axial cuts, tumour is seen filling the quadrigeminal cistern and extending ventrally around the brainstem (arrows).

tumours and rhabdoid tumours of the kidneys, and a common histogenesis may underlie both tumours. Theories of histogenesis to this point have suggested mesenchymal, neuroectodermal, or possibly a histiocytic origin. .7.14 $^{2}$ These are largely based on available morphologic, immunohistochemical, and ultrastructural analyses comparing results to other defined CNS tumour groups and normal tissues.

It should be kept in mind that the ability to infer histogenesis from immunohistochemistry and ultrastructural analysis is limited as these features reflect differentiation as well as cell of origin. More specific histogenetic evidence may come from chromosomal and gene analysis linking specific genetic abnormalities to cell lines. Preliminary data have shown monosomy of chromosome 22 in four CNS malignant rhabdoid tumours, similar to what has been observed in the majority of meningiomas. ${ }^{15,16}$ In addition, MRTs show immunohistochemical positivity for vimentin and epithelial markers such as epithelial membrane antigen and cytokeratin. This is similar to meningiomas and their cell of origin, the meningothelial or arachnoidal cell. Thus, both meningothelial cells and cells of MRTs have dual mesenchymal and epithelial differentiation. Meningothelial cells may be analogous to mesothelial serosal cells which are found in peri-renal spaces. This could represent the basis of a histogenetic link between CNS and renal MRTs.

There is little evidence supporting a neuroectodermal cell of origin. MRTs show little morphologic or ultrastructural resemblance to primitive neuroectodermal tumours (PNETs) or primitive embryonal tumours of the CNS. The reported cases of MRT have failed to show any specific neuronal or glial differentiation and immunohistochemically are negative when stained with antisera to synaptophysin, S-100, neuron specific enolase, and neurofilaments in contradistinction to PNETs. ${ }^{10,15}$ Further analysis with antisera to nestin would be of interest. Nestin is a recently described intermediate filament found primarily during early neuroectodermal development. ${ }^{17,18}$ The evidence for a histiocytic cell of origin is weak, based on scanty immunohistochemical positivity for lysozyme, and should probably be disregarded. ${ }^{19}$ Although it has been suggested that MRTs represent a diverse phenotypic "grab-bag" of tumours, ${ }^{20}$ recent studies based on the results of an expanded panel of immunohistochemical markers refutes this statement. CNS MRTs form a consistent tumour group with uniform biological behaviour, morphology and immunohistochemical profile. Although its histogenesis remains uncertain, the origin of the CNS MRT is most consistent with a tumour arising from a primitive meningothelial cell. ${ }^{10.21}$

In terms of the treatment of our patient, the child was too young to undergo cranial irradiation. Accordingly, post-operative chemotherapy was employed in an attempt to control tumour regrowth. Cycles of chemotherapy consisting of ifosphamide, carboplatin, and etoposide (so-called ICE chemotherapy) were used. Despite chemotherapy, the tumour regrew extremely rapidly at the original site, and retumed to its original size within three months. In other cases in which chemotherapy was used, it also appeared ineffective. ${ }^{8.11 .12}$ Other combinations of chemotherapy agents which have been used include intrathecal methotrexate with intravenous nitrogen mustard and vincristine, ${ }^{12}$ cyclophosphamide with vincristine, cisplatin and etoposide, ${ }^{12}$ cis-platinum with infusion of adriamycin ${ }^{11}$ and the 8 drugs in 1 day regimen formulated by the International Society of Paediatric Oncologists. ${ }^{8}$

In several cases in which high doses of radiation were used (> 5000 cGy total), tumour growth was partially inhibited. ${ }^{11,12}$ Agranovich et al. report a case in which tumour regrowth was reported to have been limited to areas outside of the field of the radiotherapy. "They suggest that CNS dissemination of the tumour may be inhibited by radiation of the complete craniospinal axis. However, despite all forms of treatment, these 
tumours have usually been uniformly and swiftly fatal. Clearly, newer treatment strategies are desperately needed to halt the relentless progression of this uncommon childhood brain tumour.

\section{REFERENCES}

1. Beckwith JB, Palmer NF. Histopathology and prognosis of Wilm's tumour. Results from the first national Wilm's tumour study. Cancer 1978; 41: 1937-1948.

2. Haas JE, Palmer NF, Weinberg AG, Beckwith JB. Ultrastructure of malignant rhabdoid tumour of the kidney. Hum Pathol 1981; 12 : 646-657.

3. Beckwith, JB. Wilm's tumour and other renal tumours of childhood: a selective review from the National Wilm's tumour study pathology center. Hum Pathol 1983; 14: 481-492.

4. Briner J, Bannwart F, Kleihues $P$, et al. Malignant small cell tumour of the brain with intermediate filaments - a case of a primary cerebral rhabdoid tumour. Pediatr Pathol 1985; 3 (abstr): 117-118.

5. Sotelo-Avila C, Gonzalez-Crussi F, deMello D, et al. Renal and extrarenal rhabdoid tumours in children; a clinicopathologic study of 14 patients. Semin Diagn Pathol 1986; 3:151-163.

6. Kapur S, Patterson K. Primary rhabdoid tumour of the cerebellum. Pediatr Pathol 1986; 5: 110

7. Biggs PJ, Garen PD, Powers JM, Garvin AJ. Malignant rhabdoid tumour of the central nervous system. Hum Pathol 1987; 18: 332-337.

8. Jakate SM, Marsden HB, Ingram L. Primary rhabdoid tumour of the brain. Virchows Arch (A) 1988; 412: 393-397.

9. Ho PSP, Lee W-H, Chen C-Y, et al. Primary malignant rhabdoid tumour of the brain: CT characteristics. J Comput Assist Tomogr 1990; 14: 461-463.
10. Chou SM, Anderson JS. Primary CNS malignant rhabdoid tumour (MRT); report of two cases and review of literature. Clin Neuropathol 1991; 10: 1-10.

11. Agranovich AL, Ang L-C, Griebel RW, et al. Malignant rhabdoid tumour of the central nervous system with subarachnoid dissemination. Surg Neurol 1992; 37: 410-414.

12. Hanna SL, Langston JW, Parham DM, Douglass EC. Primary malignant rhabdoid tumour of the brain: clinical, imaging, and pathologic findings. Am J Neuroradiol 1993; 14: 107-115.

13. Velasco ME, Brown JA, Kini J, Ruppert ES. Primary congenital rhabdoid tumour of the brain with neoplastic hydranencephaly. Child's Nerv Syst 1993; 9: 185-190.

14. Gonzalez-Crussi F, Goldschmidt RA, Hsueh W, et al. Infantile sarcoma with intracytoplasmic filamentous inclusions: distinctive tumour of possible histiocytic origin. Cancer 1981; 49:2365.

15. Berry PJ, Vujanic GM, Malignant rhabdoid tumour. Histopathology 1992; 20: 189-193.

16. Biegel JA, Burk CD, Parmiter AH, Emanuel BS. Molecular analysis of a partial deletion of $22 \mathrm{q}$ in a central nervous system rhabdoid tumour. Genes Chromos Cancer 1992; 5: 104-108.

17. Dahlstrand J, Collins VP, Lendahl U. Expression of the class VI intermediate filament nestin in human central nervous system tumours. Cancer $\operatorname{Re} 1992$; 52: 5334-5341.

18. Dahlstrand J, Zimmerman LB, McKay RDG, Lendahl U. Characterization of the human nestin gene reveals a close evolutionary relationship to neurofilaments. J Cell Science 1992; 103: 589-597.

19. Lemos LB, Hamoudi AB: Malignant thymic tumour in an infant (malignant histiocytoma). Arch Pathol Lab Med 1978; 102: 84.

20. Weeks DA, Beckwith JB, Mierau GW. Rhabdoid tumour: an entity or phenotype? Arch Pathol Lab Med 1989; 113: 113-114.

21. Bonnin JM, Rubinstein LJ, Palmer NF, Beckwith JB: The association of embryonal tumours originating in the kidney and in the brain. Cancer 1984; 54: 2137-2146. 